\title{
Journal of Nanotechnology and Materials Science
}

\section{Nanotechnology Based Virosomal Drug Delivery Systems}

\author{
Nagi F. Idris ${ }^{1}$, Ramchandra N. Chilkawar ${ }^{2}$, Basavaraj K. Nanjwade ${ }^{3 *}$, Teerapol Srichana ${ }^{4}$, Azam Sharif \\ Md. Shafioul ${ }^{5}$ \\ ${ }^{1}$ Department of Pharmacology, Faculty of Pharmacy, Omer Al-Mukhtar University, Al-Bayda, P. O. Box 919, Libya. \\ ${ }^{2}$ Department of Quality Assurance, Remidex Pharma Pvt Ltd., $2^{\text {nd }}$ Stage, Peenya Industrial Area, Bengaluru-560058, Karnataka. India. \\ ${ }^{3}$ Department of Pharmaceutics, Faculty of Pharmacy, Omer Al-Mukhtar University, Tobruk, P. O. Box 919, Libya. \\ ${ }^{4}$ Drug Delivery System Excellence Centre, Faculty of Pharmaceutical Sciences, Prince of Songkla University, Hat-Yai-90112, Songkla, Thailand. \\ ${ }^{5}$ Chemical Kinomics Research Center, Future Convergence Research Division, Korea Institute of Science and Technology (KIST), Seoul 136-791, \\ South Korea.
}

\begin{abstract}
Over the years there has been a great revolution in drug delivery technologies. Virosomes delivery is an example of the various novel drug delivery systems available. Virosome is a drug or vaccine delivery mechanism which is spherical and consisting of unilamellar phospholipid bilayer vesicle with a mean diameter is in range of 120-180 nm. Virosomes represent reconstituted empty influenza virus envelopes, which contain $70 \%$ phosphatidylcholine and remaining $30 \%$ neuraminidase (NA) and haemagglutinin (HA) glycoproteins. Various techniques are used for the loading the drug, protein and peptide like package inside the IRIV (immunopotentiatin reconstituted influenza virosome), intrigrated into lipid bilayer also anchored into lipid bilayer, crosslinked with Hemaglutinin (HA) and adsorbed to the membrane. Various protein, peptide, and malarial drugs are loaded in virus to deliver at particular site to give the targeted drug delivery. The prospect of drug delivery and targeting using virosomes is an interesting field of research and development. Virosomes are biodegradable, biocompatible, nontoxic, and non-autoimmunogenic attempts were made to use them as a vaccines or pharmaceutical adjuvants as well as delivery systems for pharmaceutical drugs, nucleic acids, genes for therapeutic application. Virosomes could also be exploited as carriers for targeted drugs and for immunomodulating molecules particularly in cancer therapy. The success of virosomal drug delivery depends on the methods used to prepare the encapsulated bioactive materials and incorporate them into the virosomes, characterization and formulation of the finished preparation. This review article gives an insight of virosomes as a newer method of drug delivery and futuristic tool.
\end{abstract}

Keywords: Virosomes; Biocompatible; Biodegradable; Drug delivery; Gene delivery

Abbreviations: NA: Neuraminidase; HA: Haemagglutinin; IRIV: Immunopotentiatin reconstituted influenza virosome; PL: Phospholipids; PC: Phosphatidylcholine; APCs: Antigen presenting cells; CTLs: Cytotoxic T lymphocytes; PAMP: Pathogens-associated molecular patterns; TAA: Tumour Associated Antigens; CSP: circumsporozoite protein.

*Corresponding author: Basavaraj K. Nanjwade, Professor of Pharmaceutics, Department of Pharmaceutics, Faculty of Pharmacy, Omer Al-Mukhtar University, Tobruk, P. O. Box 919, Libya. Tel: 00218944828793; E-mail: nanjwadebk@gmail.com

\section{Received date: Sep 19, 2014 Accepted date: Dec 11, 2014 Published date: Dec 15, 2014}

Citation: Basavaraj, K.N., et al. Nanotechnology Based Virosomal Drug Delivery Systems. (2014) J Nanotech Mater Sci 1(1): 27-35.

\section{Introduction}

Virosome can use as a drug or vaccine delivery mechanism consisting of unilamellar phospholipid bilayer vesicle incorporating virus derived proteins to allow the virosomes to fuse with target cells and tissues. Virosomes are not able to replicate but are pure fusion-active macro and nano level vesicle. These are reconstituted viral envelopes that can serve as vaccines and as vehicles for cellular delivery of macromolecules and nanomolecules. The prospect of drug delivery system and targeting cells and tissues using virosomes is an interesting field of research and development. Virosomes are biodegradable, biocompatible, nontoxic and non- autoimmunogenic attempts were made to use them as a vaccines or adjuvant as well as delivery systems for drugs, nucleic acids, genes for therapeutic application. Influenza virus is the most common virus for choice of use. The success of virosomal drug delivery systems depends on the various methods used to prepare the encapsulated bioactive materials and incorporate them into the virosomes, characterization and formulation of the finished virosomal preparation. Virosomes protect pharmaceutically active substances from proteolytic degradation and low $\mathrm{pH}$ of endosomes and allowing their contents to remain intact when they reach the cytoplasm ${ }^{[1]}$.

The transfer of genes to target cells by various delivery vehicles are mainly divided into viral and non-viral vectors systems. Viral vectors are obtained by replacement of one or more viral genes by a gene of interest and are considered to be the most efficient transducing systems ${ }^{[2,3]}$. Efficiency relates to properties of the viral capsid proteins or membrane glycoproteins, the ability to bind to cellular receptors and to pass through or

Copy rights: (C2014 Basavaraj K.N. This is an Open access article distributed under the terms of Creative Commons Attribution 4.0 International License. 
fuse from/with cellular membranes. But the safety of viral vector system remains a matter of major problems and issues related to insertion mutagenesis as observed with retroviruse ${ }^{[4]}$ and the induction of undesirable immune responses and inflammation still pose major challenges ${ }^{[5,6]}$. For non-viral gene delivery, chemical approaches (e.g. cationic polymers, cationic lipids and nanoparticles) and physical methods (e.g. gene gun and electroporation) are being employed ${ }^{[7]}$. Cationic liposomes are the most extensively studied vehicles ${ }^{[8-11]}$. In these systems, cationic lipids condense DNA through electrostatic interactions with the negatively charged phosphate groups of the nucleic acid, thereby forming so-called lipoplexes. With respect to in vivo use, liposomal delivery systems combining the characteristics of cellular interaction of viral vectors with the safety of liposomal delivery systems. Virosomes are the reconstitutes viral envelopes, having lipid membranes, viral spike glycoproteins, but absence of viral genetic material. Externally virosomes resemble that of a virus particle, with spiky proteins protruding from their membrane, and their interior compartment is empty. Almeida et al... Were the first to prepare virosomes, who inserted purified influenza spike proteins into preformed liposomes ${ }^{[12]}$. Virosome technology is developed in order to overcome the problem of incomplete delivery to targeting cells, tissues and organs of the body. The new generation of therapeutics against cancer or neurodegenerative disorders which require the delivery system that target drugs to specified cell types and host tissues by receptor-mediated uptake and controlled release.

Thus, the virosomal technique represents a novel sophisticated delivery system to meet all the above challenges and drawbacks. There after a wide range of viral envelopes have been reconstituted, including those of sendai virus and sindbis virus $^{[13]}$. Virosomes display viral envelope of glycoproteins and their native conformation stimulate humoral responses. The receptor-binding and membrane-fusion properties of the viral envelope of glycoprotein can be preserved and virosomes can be used as transport vehicles for cellular delivery of biologically active macromolecules and nanomolecules.

Virosomes protect pharmaceutically active substances from proteolytic degradation and low $\mathrm{pH}$ of endosomes and allowing their contents to remain intact when they reach the cytoplasm. These are major advantage of virosomal carrier systems over other than drug-delivery vehicles and adjuvant including liposomal and proteoliposomal carrier systems.

\section{What is Virosomes}

Virosomes are small spherical vesicles containing viral membrane proteins (fusion proteins of the influenza virus) embedded in their lipid membranes. Diameter is in range of 120-180 nm. Virosomes represent reconstituted empty influenza virus envelopes and devoid of the nucleocapsid including the genetic material of the source virus. Virosomes are not posible to replicate but are pure fusion-active vesicles. The main constituents of Immunostimulating Reconstituted Influenza Virosomes (IRIVs) consist of naturally occurring phospholipids (PL) and phosphatidylcholine (PC). Phosphatidylcholine forms approximately $70 \%$ of the virosomal structure. The remaining $30 \%$ of membrane components are the envelope phospholipids originating from the influenza virus that provide neuraminidase (NA) and haemagglutinin (HA) glycoproteins (Figure 1).
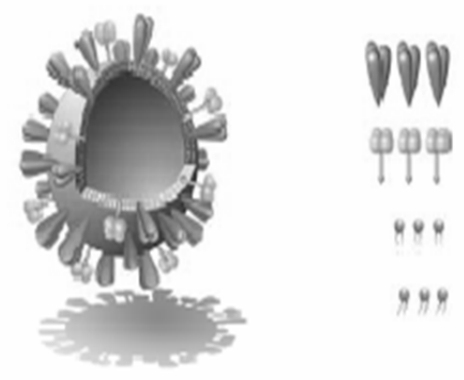

Hemagglutinin

Neuraminidase

Phosphatidylcholine

Phosphatidylethanolamine

Figure 1: Ultrastructure of a Virosome: Influenza virus envelope with functional viral envelope of glycoprotein.

The neuraminidase (NA) is a tetramer composed in four equal spherical subunits hydrophobically embedded in the IRIV (Immunostimulating Reconstituted Influenza Virosomes) membrane by a central stalk and can readily intercalate into the phospholipid membrane. Neuraminidase (NA), present on the IRIV's (Immunostimulating Reconstituted Influenza Virosomes) surface and aids its action by the same mechanisms through which it enhances influenza virus pathogenicity, it catalyses the cleavage of $\mathrm{N}$ acetylneuraminic acid (sialic acid) from bound sugar residues resulting in a decreased viscosity of the host's mucus and allowing to the influenza virus an easier access to epithelial cells (Figure 2).

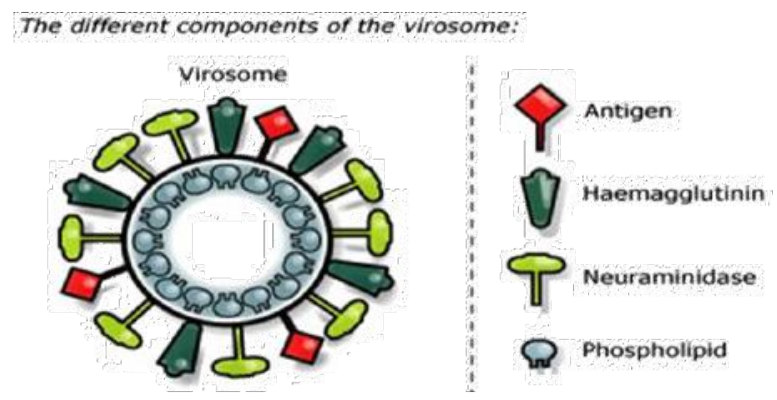

Figure 2: Showing different components of virosomes.

The same process leads to destruction of the HA (haemagglutinin) receptors within the cell membrane to which viruses and IRIVs bind. This allows the virus particles to avoid aggregation, as newly formed virus particles do not adhere to the infected host cell membrane after budding and allowing the influenza virus to retain its mobility. The mode of action of IRIVs is dependent on the influenza haemagglutinin. Haemagglutinin, intercalated into the phospholipid bilayer, stabilises the liposome base by preventing fusion with other liposomes. The HA (haemagglutinin), the major influenza antigen, is formed from two polypeptides, HA1 and HA2, that are responsible, upon conformational change, for the fusion of the virus with the endosomal membrane. The HA1 globular head contains a receptor site that has a high affinity towards sialic acid, present on the surface of antigen presenting cells (APCs), such as macrophages and lymphocytes and therefore facilitates the binding of IRIVs (Immunostimulating Reconstituted Influenza Virosomes). The actual fusion of IRIVs (Immunostimulating Reconstituted Influenza Virosomes) with the endosomal membranes is then mediated by the HA2 polypeptide ${ }^{[14-18]}$. 


\section{Need of Virosomal Technology}

State-of-the-art-drugs must meet diverse medical, pharmacological and pharmaceutical demands. They have to be efficient, reliable and reduced severe side-effects. Market aspects also need to be considered. Due to the high number of competing products on the market, drugs should be cost effective. Stability is an issue as well, because drugs are often subject to unfavourable delivery and storage conditions. Moreover, the market potential of a drug also rises with its compliance and convenience. Apart from these requirements novel drug delivery system technologies should provide improved bioavailability, minimizing the quantities of the drug needed to be taken by the patient. Several drugs in clinical tests are discontinued at various stages in the development process because of the lack of suitable delivery technologies. Drug delivery system may rescue some of the products by overcoming these difficulties. The new generation of therapeutics against cancer or neurogenerative disorders needs drug delivery systems which guarantee targeted drug delivery to specified host tissues and cells, receptor-mediated uptake and controlled release into the lumen of the cells. This all requires vision and long-term, challenges meet with the development of a highly sophisticated carrier system based on its proprietary virosomal technology.

Effective vaccination against infectious agents or tumour antigens requires the induction of potent humoral and cellular immune responses based on antibodies and CD4+ T helper cells or cytotoxic T lymphocytes (CTLs). Depending on the targeting and the induction of neutralizing antibodies is also very important. There is a need for antigen delivery systems capable of directing nonreplicating exogenous antigen into the $\mathrm{MHC}$ class II and I presentation pathway Virosomes can be used as delivery system for unrelated molecules in vitro and in vivo and a list of so far tested applications of virosomes as carrier of different products (Table 1). The route of immunization is another important feature and which characterizes this system. Virosomes can be administered via nasal and vaginal mucosal, intradermal and intramuscular routes. Stimulation of local and systemic immune responses may improve the immunogenicity and efficacy of the vaccine. The first line of defence for the host is mucosal immunity, which is a major component of the immunological humoral and cell mediated response. Prevention and treatment of infectious diseases and active specific cancer immunotherapy frequently require the induction of a powerful cellular immune response, which can be achieved with virosomes carrying different types of antigens, such like peptides, proteins, virions or molecules such as an oligonucleotides or plasmids. An attractive feature of virosomes is that during the reconstitution process a variety of molecules can be incorporated either in the virosomal membrane or in the aqueous interior. Now days in drug delivery system Chimeric Virosomes (C-IRIV) can be used as a suitable carrier system for DNA and RNA since these fusogenic particles mimic like a virus. Virosomes can also be used as adjuvant, vehicle and this property has already been validated by three licensed vaccines against hepatitis A (Epaxal, Berna Biotech) and influenza (Inflexal, Berna Biotech and Invivac, Solvay Pharmaceuticals), which combine the superior safety of a subunit vaccine with the immunogenic potential and biological properties of a virus-like structure ${ }^{[19,20]}$.
Table 1: Applications of Virosomes as carriers of different molecules

\begin{tabular}{|l|l|l|}
\hline Molecules & $\begin{array}{l}\text { Development Candidates, } \\
\text { or Research Leads }\end{array}$ & $\begin{array}{l}\text { Refer- } \\
\text { ences }\end{array}$ \\
\hline Mumps F/HN DNA plasmid & Research & {$[43]$} \\
\hline PTH-rP DNA plasmid & Research & {$[40]$} \\
\hline CEA and CD40L DNA plasmid & Research & {$[44]$} \\
\hline Antisense L-myc OPT & Research & {$[45]$} \\
\hline Small interfering RNAs & Research & {$[38]$} \\
\hline F RSV protein & Research & {$[46]$} \\
\hline Fab' Her-2 Neu and doxorubicin & Research & {$[41]$} \\
\hline mAb anti epithelial glycoprotein 2 & Research & {$[47]$} \\
\hline $\begin{array}{l}\text { Plasmodium falciparum SPf66 } \\
\text { peptide }\end{array}$ & Research & {$[14]$} \\
\hline $\begin{array}{l}\text { Plasmodium falciparum AMA-1 } \\
\text { peptide }\end{array}$ & $\begin{array}{l}\text { Phase I clinical trial (PE- } \\
\text { VION) }\end{array}$ & {$[42]$} \\
\hline HCV peptides & Research & {$[48]$} \\
\hline Amyloid Ab peptides & $\begin{array}{l}\text { In development (PEVI- } \\
\text { ON) }\end{array}$ & {$[49]$} \\
\hline
\end{tabular}

\section{Structure of Virosomes}

Virosomes are produced by dissolving the envelope of a virus by a detergent or short-chain phospholipids. Next viral genetic material and non-membrane proteins are removed. The subsequent removal of the detergent or short-chain phospholipids, the viral membrane is reconstituted and producing a virosome containing the viral membrane proteins and lipids. Viral proteins confirm to virosome based vaccine structural stability, homogeneity and increase the immunologicalproperties of prepared virosomes (Figure 3 and Figure 4) ${ }^{[21]}$.

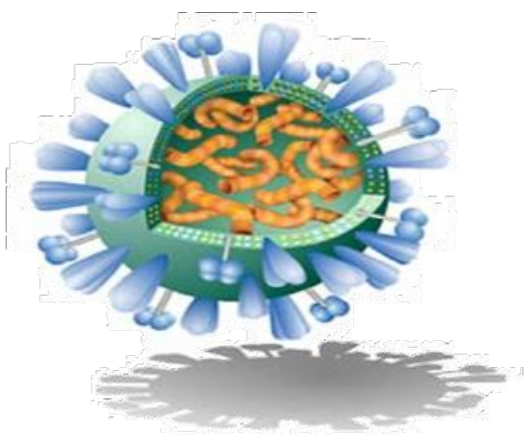

Figure 3: Influenza virus

Influenza virus shown in figure (Figure 3). The envelope made of influenza lipids constitute the membrane and proteins called Hemagglutinin (HA) and Neuraminidase (NA) are intercalated on its envelope. Inside the envelope the Nucleocapsid and the genetic material of the source virus.

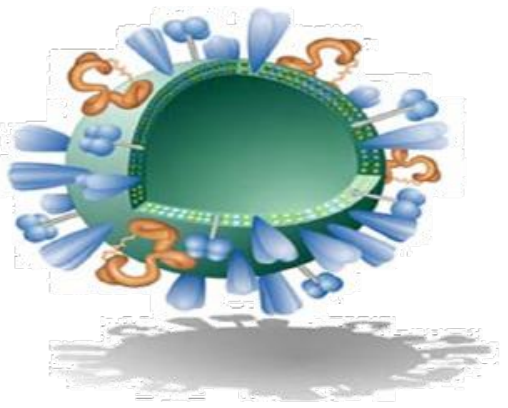

Figure 4: Lipid bilayer of virosomes. 
As shown in the (Figure 4), the virus is devoid of the nucleocapsid including the genetic material of the source virus and the virosome is created and devoid of its substance, it is not suitable to replicate, its cause an infection or a disease. Virosomes contain functional viral envelope glycoprotein's-influenza virus hemagglutinin and neuraminidase intercalated in the phospholipid bilayer membrane ${ }^{[22]}$. They have a typical mean diameter of $150 \mathrm{~nm}$ and essentially virosomes represent reconstituted empty influenza virus envelope devoid of the nucleocapsid including the genetic material of the source virus ${ }^{[23]}$.

\section{Vehicle for Vaccines}

Vaccine is a biological preparation that improves immunity to a particular infectious microorganism (microbe). A vaccine typically contains a small amount of an 'A' agent (immunogen) that resembles to a microorganisms. The immunogen stimulates to the body's immune system to recognize it as a foreign material, destroy it and "remember" it. The immune system can more easily recognize and destroy or neutralize the correspondent microorganism that is its later encounters by avoiding severe infection and subsequently its pathologies. The immunogen is made up of some parts of the microbe, as like proteins, which are seen by the immune system as the microbe itself. This immunogen usually needs a vehicle and adjuvant to be delivered efficiently in the body and body parts ${ }^{[24]}$.

\section{Virosomes Adjuvant System}

Virosomes are virus-like spherical particles with a average diameter of $150 \mathrm{~nm}$ (nanometrs), consisting of reconstituted influenza virus envelopes and lacking the genetic material of the native virus the cell binding and membrane fusion properties of the native virus also mediated by the viral envelope glycoprotein haemagglutinin. The functional characteristics of virosomes form the basis for their enhanced immunogenicity ${ }^{[25]}$. The Combination with combinatorial chemistry, this approach may have great potential for the rapid optimization of molecularly defined synthetic vaccine candidates against a wide variety of infectious agents.

\section{Properties of Virosomes}

Virosomes are biodegradable; biocompatible, and non-toxic. An antigen can be incorporated into virosomes, adsorbed to the virosome surface and integrated in to the lipid membrane, either via hydrophobic domain or lipid moieties cross-linked to the antigen. They are also being considered for HIV-1 vaccine research. Virosomes were used as a drug carrier mechanism for experimental cancer therapies ${ }^{[2]]}$.

\section{Delivery through viral vector}

Viral vectors can be utilized for epidermal transfer of the DNA or other suitable antigen. Viral vectors include adenovirus vector, RSVE and HSV amplicon vector. The epidermal gene transfer by means of HSV amplicon vector produces necrosis and cytotoxicity and hence, they cannot be applied repeatedly or for longer period of contact. Reconstituted viral vectors targeting of virosomes have also been utilized for intracellular targeting of encapsulated DNA/antigen. The RSVE can be applied topically for efficient gene or antigen transfer. RSVE constitute two types of proteins, namely haemagglutin $(\mathrm{HN})$ and fusion $(\mathrm{F})$ proteins, which determine the fusogenicity. The RSVE can be effective as cell microinjection vector to transfer immune response through topical route. They can serve as an efficient immunoadjuvant ${ }^{[27]}$.

\section{Pharmacokinetics of Virosomes}

Pharmacokinetics information can be used to interpret the differences in the pharmacological effect of liposomal-entrapped drug and free drug, subsequently can be exploited for dose designing. Pharmacokinetics deals with time course of absorption, distribution and degradation of the virosomal carriers in vivo. The pharmacokinetics of virosomes requires the knowledge of possible accessible sites after intravenous administration as this is the most accepted route for various virosomal formulations exploited for clinical therapeutics except topical formulations. Virosomes alters both the tissue distribution and the rate of clearance of a drug as they are affected by the pharmacokinetics parameters. Under optimal conditions the drug has been carried within the virosomal aqueous phase during circulation and it leaks at sufficient rate to become bioavailable on arrival at tissue or other specific sites. Bioavailability in case of virosomal carriers can be defined as the amount of free drug that is able to escape the confines of the carrier and thus become available for redistribution to neighboring tissue ${ }^{[28]}$.

\section{Mechanism of Action of Virosomes}

Virosomes act both as a drug delivery carrier and also as an adjuvant with multiple functions during the induction of an immune response to bodies. The carrier function comprises the positive effects of embedding the antigen into a higher structure, the virosome particle. The adjuvant function relates to immunestimulating properties of the virosomes and their components on the immune system. Most importantly, virosomes succeed in stimulating specific immunity without causing nonspecific inflammation $^{[29]}$. By this mechanism, an antigen-specific helper immune response is induced, which potentiates both the cellular and humoral immune response (Figure 5).

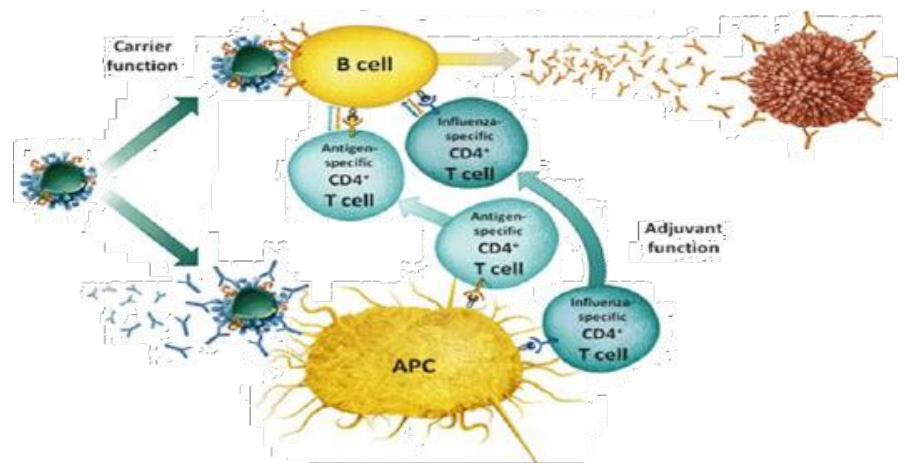

Figure 5: Mode of action of virosomes

\section{Carrier Function Response}

The integration of the antigen into the higher structure of the virosome particle stabilizes the antigen to preserves the native status of $B$ cell epitopes and protects the antigen from degradation. The antigen displayed on the surface virosomals which mimics the original pathogen or target cell and thereby favours the generation of antibodies relevant for protection. The presentation of the antigen as a repetitive surface structure enhances its recognition by antibody-producing B-cells. The size and surface structure of the virosome particles make them an 
attractive target for uptake and processing by immune cells and which is a crucial step in the initiation of an immune response $\mathrm{e}^{[30]}$.

\section{Virosomes Adjuvant Function}

The adjuvant function of virosomes relies on the presence of influenza-derived envelope proteins, in particular the predominant haemagglutinin (HA). Pre- existing antibodies against influenza bind to virosomes and tag them efficiently for rapid uptake and processing by antigen presenting cells (APC). The natural function of antibodies is to bind virus and block infection. Pre-existing influenza-specific helper T cells are activated by those antigen presenting cells (APC) displaying the processed fragments of the influenza proteins. Influenza proteins activated helper cells rapidly proliferate and secrete cytokines to support and enhance the induction of effect or immune cells, e.g. antibody-producing cells ${ }^{[31]}$.

\section{Difference between Virosomes and Liposomes}

Viral envelope glycoproteins with receptor-binding and membrane-fusion properties that enable the cellular liposomes have been considered promising vehicles for targeting and delivery of biologically active molecules to living cells both in vitro and in vivo. Liposomes have little potential to fuse with cells and generally fail to provide appreciable delivery of encapsulated molecules to the cell cytoplasm. Virosomes containing functional delivery of encapsulated molecules ${ }^{[32]}$.

\section{Virosome uptake by Cells}

Entry of virosomes into target cells divided into two different steps:

a. Attachment: This involves binding of the virosomes via HA to the cell receptors that are a membrane glycoprotein or glycolipid with terminal sialic acid. In case of specific virosomes, Fab fragments are coupled by a cross-linker with a spacer arm to the virosomal surface. Specific virosomes will additionally recognize antigenic structures on the targeting cell surface and resulting in an attachment to target cells by two different binding mechanisms. Thus, specific virosomes exert selectivity for special cell types.

b. Penetration: After attachment entry of virosomes occurs by receptor-mediated endocytosis. The virosomes are trapped in endosomes, acidic fusion of the virosomal membrane with endosomal membrane. The fusion is mediated by the viral spike glycoprotein hemagglutinin (HA). The membrane-fusion reaction in the endosome librates the virosomes from its lipid envelope and provides access for the encapsulated drugs to the cytosol.

\section{Functions by the Carrier}

The integration of the antigen into the higher structures of the virosomes particle stabilize the antigens, preserves the native status of $B$ cell epitopes and protects the antigens from degradation. Moreover, the presentation of the antigen as a repetitive surface structure enhances its recognition by antibody-producing B cells.

\section{Memory Support}

The presence of influenza-derived hemagglutinin (HA) provokes a memory response as a vast majority of people have a degree of natural, pre- existing immunity against influenza. This comprises both humeral and cellular immunity: pre-exist- ing influenza-specific antibodies tag virosomes efficiently for rapid uptake and processing by antigen presenting cells (APC). Memory $\mathrm{T}$ helper cells rapidly proliferate and secrete cytokines to support and enhances the induction of effector immune cells.

\section{Method of Preparation of Virosome}

\section{a. Selection of virus for virosomes}

Virosomes are reconstituted viral envelope that can be derived from different virus. Influenza virus envelope is most often used to produce virosome but virosome can also be made from Sendai virus, Epstein-burr virus, HIV, sindbis, semliki-forest virus, friend murine leukaemia virus, herpes simplex virus, Newcastle disease virus ${ }^{[29,33-35]}$.

\section{b. Selection of antigen for virosomes}

Antigen is selected as per our requirements. Antigens such a parasite, carcinogenic cell, bacterium or whole cell is used as antigens. Cell components such as DNA, RNA or plasmid can also be used as antigen. These antigens are coupled to lipid anchor so antigen will be ready to load on virosome (Figure 6).

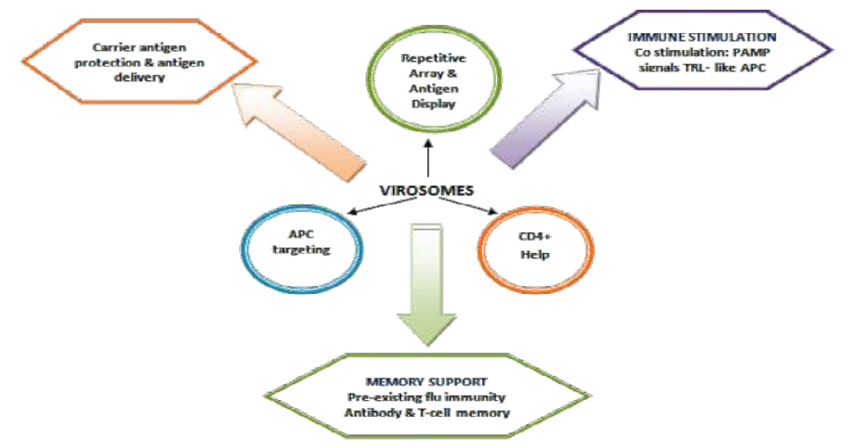

Figure 6: Showing activation of antibody through virosomes.

\section{c. Reconstitution of virosome}

Virosome solubilized with detergent of octaglucoside, triton $\mathrm{X}-100$, nonidert $\mathrm{p}-40$. Because of solubilization with detergent internal viral protein and genetic material will Sediment then detergent is removed by different method such as dialysis and hydrophobic resins from supernatant. Then using ultracentrifugation process viral matrix protein and nucleiocapsid is removed. Viral phospholipids (82\%) and viral protein is recovered in supernatant. Protein obtained in supernatant represents viral envelope protein only. Now antigen which is already coupled to lipid anchor is mixed with polymer or surfactant solution and this solution is processed with virosome carrier so that antigen bound virosome is obtained (Figure 7).

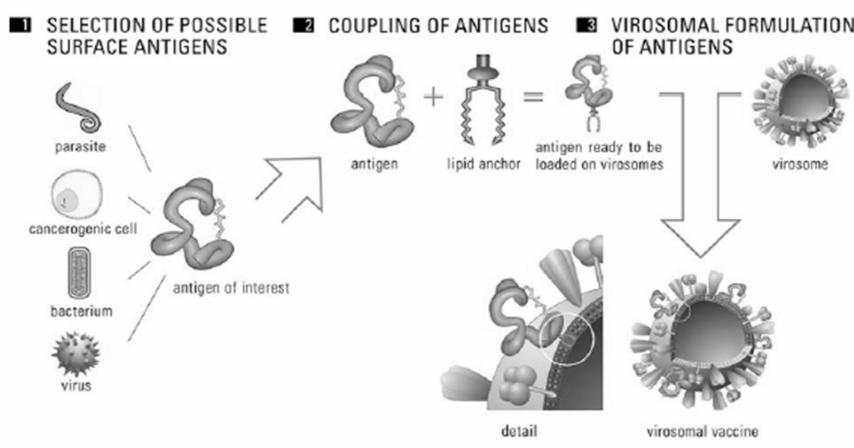

Figure 7: General method for preparation of virosomes. 


\section{Characterization of Virosomes}

\section{a. Protein detection}

Preparation of virosome generally result in a relatively uniform protein-to-lipid ratio, sodium dodecyl sulfate polyacrylamide gel electrophoresis (SDS-PAGE) can confirm the presence of hemagglutinin (HA) protein in the virosomes ${ }^{[33]}$.

\section{b. Structure and size}

Negative-stain electron microscopy can generally be used to determine the ultra structure and different size of virosomes. The staining solutions should be neutral $\mathrm{pH}$, to avoid acid-induced conformational changes of hemagglutinin (HA) ${ }^{[36]}$.

\section{c. Fusion activity}

Generally virosomes exhibit $\mathrm{pH}$-dependent membrane fusion activity similar to native influenza virus. Virosomal fusion along with biological or artificial target membrane is visualized with a fluorescent resonance energy transfer assay (RET). Alternatively fusion can be assessed in vitro with an excimer assay using pyrene-labeled lipids and decrease of surface density of the pyrene-phosphatidylcholine-label on fusion with an unlabeled membrane corresponds to a reduction in excimer fluorescence ${ }^{[35]}$.

\section{Route of Administration of Virosomes}

The virosomes are administered in a variety of parenteral routes including intravenous (IV), intramuscular (IM), subcutaneous (SC), intra-arterial and inhalable delivery. In addition, virosomes can be administered topically, orally or transdermally. The virosomes also can be incorporated into implantable devices for long-term release ${ }^{[37]}$.

\section{Virosome for Drug Targeting System}

The major problem of combining chemotherapy and immunotherapy is the severe side effects that limit the use of doxorubicin as a cytotoxic drug. We can use virosomes (reconstituted fusion-active viral envelopes) as a new drug delivery system and have shown that virosomes are capable of binding and penetrating into tumour cells and delivering cytotoxic bioactive drugs. We have additionally demonstrated that conjugating Fab' fragments of an anti-rNeu monoclonal antibody $(\mathrm{mAb})$ to virosomes selectively and efficiently inhibits tumour progression of established rNeu over expressing breast tumours. Fab'-Doxo-Virosomes combine the antiproliferative properties of the $\mathrm{mAb}$ and the cytotoxic effect of doxorubicin in vivo. Fab'-Doxo-Virosomes significantly inhibit tumor formation at a tumor load representing metastatic spread. The results indicate that virosomes conjugated with an antibody against a tumour antigen are a promising new selective drug delivery system for the treatment of tumours expressing a specific tumour antigen ${ }^{[38]}$.

\section{Novel Drug-Delivery Approaches}

Virosomes are particularly useful for delivering nucleic acids or genes. These compounds are delivered into the host cell cytoplasm on fusion of the virosome with the endosome or cell plasma membrane. The nucleic acids or genes encoding a naturally occurring protein can be introduced into host cells and can be expressed, provided that the expression cassette contains the proper cis-acting regulatory elements. Drugs or nucleic acids can be incorporated into the virosome at the time of virosome targeting preparation. The bioactive compound is typically added to the lipid-HA-containing solution following removal of the nucleiocapsid. Alternatively, the bioactive compound is initially incorporated into a liposome, which is then fused with a virosome containing two haemagglutinin with different $\mathrm{pH}$ thresholds to form a virosome and liposome hybrid. Proteins also can be delivered to cells via virosome. For example, the gelonin subunit 'A' of diphtheria toxin and ovalbumin has been successfully delivered by virosome to target cells ${ }^{[31]}$ (Figure 8 ).

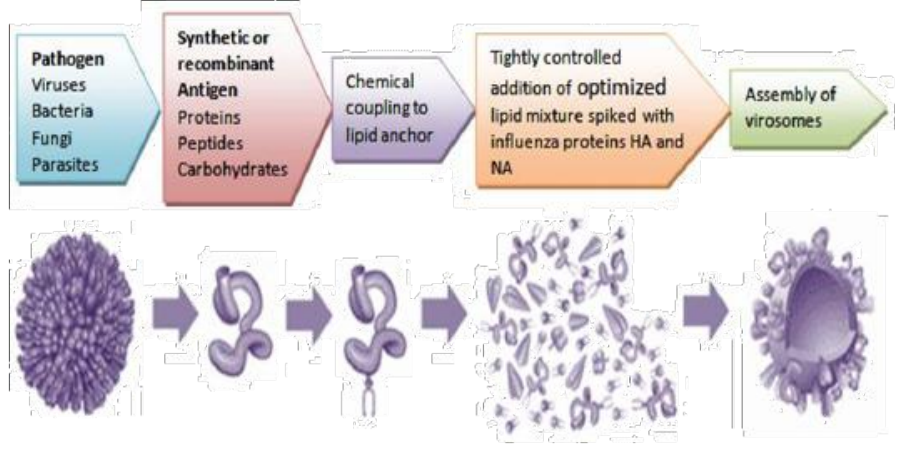

Figure 8: Showing assembly of virosome in a controlled manner.

\section{Merits of Virosomal Drug Delivery}

1. Virosomal technology is approved by the FDA for use in humans and it has a high Safety profile.

2. Virosomes are nontoxic, biodegradable and biocompatible.

3. No disease-transmission risk

4. No auto immunogenity or anaphylaxis.

5. Broadly applicable with almost all important bioactive drugs enables drug delivery into the cytoplasm of target cell.

6. Promotes fusion activity in the endolysosomal pathway.

7. Protects drugs against degradation.

8. Target-specific delivery of antigens and amplification of the immune response.

9. Extended uptake, distribution and elimination of drug in body.

10. Promote fusion activity in endosomal pathway.

11. Virosome allow patient specific modular vaccine regimen.

12. Up scaling according to standard procedure.

13. The fully functional fusion-activity of virosomes enables receptor mediated uptake and natural intracellular of the antigen, which leads to the stimulation of immune system: humoral and cellular immune responses.

14. The antigen is partially protected from extra cellular degradation and the resulting depot effect greatly facilitates immune potentiation $^{[31]}$.

\section{Applications of Virosomes \\ Virosomes in Gene Delivery System}

Haemagglutinin membrane fusion protein of influenza virus is known to mediate a low-pH- dependent fusion reaction between the viral envelope and the limiting membrane of the endosomal cell compartment following cellular uptake of the virus particles by receptor- mediated endocytosis here, they exploited this activity of haemagglutinin to achieve efficient gene delivery to cultured cells ${ }^{[39]}$.

\section{Virosomes in RNA/DNA Delivery System}

Small interfering RNA (siRNA) encapsulated in virosomes are able to down regulate the synthesis of newly induced and constitutively expressed proteins and overcoming the lack of suitable delivery methods for these molecules. Administration route is Intraperitoneal injection of siRNA loaded virosomes resulted in delivery of the nucleotides to cells in the peritoneal 
cavity ${ }^{[40]}$.

\section{Virosomes Activation of Murine Lymphocyte}

The incorporation of Salmonella Minnesota rough-LPS in liposome's reduced the potency of LPS to stimulate splenocyte proliferation and cell surface kappa-light chain expression on $70 \mathrm{Z} / 3$ pre-B cells by over 100 -fold. The Salmonella Minnesota rough-LPS inserted into virosomes was at least 10 -fold more potent than free LPS, when both prebound virosomes were allowed to be taken up by the cells at neutral $\mathrm{pH}$ and when the virosomes were fused into the plasma membrane by low $\mathrm{pH}$ treatment. Virosomal LPS remained 2- to 10-fold more potent than free LPS in stimulating B lymphocytes and at least 100fold more active than liposomal LPS or fusion inactivated virosomes. After low $\mathrm{pH}$-induced fusion with the plasma membrane, the majority $(80 \%)$ of the prebound virosomes had fused with the cells, compared with about $8 \%$ after neutral uptake ${ }^{[41]}$.

\section{Virosomes for Cancer Treatment}

Virosomes have been also used in the oncology area to carry peptides corresponding to Tumour Associated Antigens (TAA), as in the case of peptides derived from the parathyroid hormone- related protein (PTH-rP) or from recombinant proteins such as Her-2/neu. Fab'-Doxo- virosomes combined the anti-proliferate properties of the monoclonal antibody and the cytotoxic effect of doxorubicin in vivo. Fab'-Doxo-virosomes significantly inhibited tumour formation at a tumour load of metastasis spread, indicating that this can also be considered to be a promising new selective drug delivery system for the treatment of tumours expressing a specific tumour antigen. The immunogenic effect of the HER-2/neu protein was significantly increased when the protein was linked to virosomes; they are encapsulated as a soluble protein or bound to a virosomal membrane. Virosomes elicited humoral and cell-mediated responses against TAA, as well as inducing tumour rejection ${ }^{[42]}$.

\section{Virosomes for Malaria Therapy}

Malaria vaccine, containing virosome-formulated anti-malaria peptides, shows a good tolerability and a highly specific immune response in humans. Scientists have identified and optimized synthetic peptide structures that mimic the surface loops of two candidate malaria vaccine antigens, the NPNA repeat region of the circumsporozoite protein (CSP), loop I of domain III of merozoite apical membrane antigen-1(AMA-1). Lead structures of additional antigens have been identified and additional optimised mimotopes are being developed. It is assumed that there is a need to add further mimotopes to produce an effective multistage, multicomponent malaria vaccine formulation $^{[43-51]}$.

\section{Immune Stimulation}

In addition to the influenza-specific antigens, virosomes provide pathogens-associated molecular patterns (PAMP) that deliver co-stimulatory signals to APC, which leads to a TLRlike activation ${ }^{[52,53]}$.

\section{Possible toxicity of virosomes}

Virosome mediated delivery has low toxicity and high immunogenicity with various prospective applications for the treatment and prevention of cancer, neurodegenerative disorders and infectious diseases. The toxicity of cationic formulations might be ascribed to the cationic lipids themselves, and several studies have investigated the correlation between the chemical structure of lipids and their toxicity profile ${ }^{[54]}$.

\section{Safety of Virosomes}

So-called virosomes has been developed by incorporating the HA from an influenza virus A strain into liposomes composed of phosphatidylcholine. The influenza virus surface glycoprotein HA guides the virosomes specifically to antigen-presenting cells and leads to fusion with their endosomal membrane. Process provides optimal processing and presentation of the antigens to immunocompetent cells and T lymphocytes are activated to produce cytokines. The B lymphocytes to form large amounts of specific antibodies. Stimulation of B lymphocytes also occurs through direct contact with the antigen-virosome complex. When tested for safety and immunogenicity in elderly nursing home residents in comparison to whole-virion and subunit vaccines and the virosome-formulated influenza vaccine was found to be superior with regard to reactogenicity and immunogenicity. This trivalent virosome influenza vaccine (Inflexal Berna V) is currently licensed in several European countries (e.g., Switzerland since 1997 and Italy since 1998). We investigated the safety and comparative immunogenicity of this new virosome influenza vaccine in comparison with a standard subunit influenza vaccine for a paediatric group at risk, i.e., children and adolescents with $\mathrm{CF}^{[55]}$.

\section{Targeting of Virosomes Influenza Vaccine}

Influenza is a potentially serious disease in very young children due to no background immunity in the elderly due to innate decreased resistance to infections and a poorly functioning immune system, and in individuals with an underlying disease which may render them unable to handle infections. Patients with chronic pulmonary dysfunction such as cystic fibrosis (CF) are at a particularly high risk for acquiring a severe influenza infection. Influenza disrupts the normal defense system of the respiratory tract and may lead to secondary bacterial pneumonia. An Influenza vaccination has been shown to be beneficial to children's, healthy working adults and elderly subjects with or without risk factors. Therefore, many public health authorities recommend routine annual immunization of high-risk individuals. In many countries, including Switzerland, targeted vaccinations are reimbursed by health insurance or by public funds. Despite these recommendations and incentives, at most half of Swiss citizen's $\geq 65$ years old are immunized annually against influenza. Recent surveys on influenza vaccination coverage showed these figures to be higher in France ( $\geq 70 \%$ ), in The Netherlands (58 to $64 \%$ ), and in the United States (55 to 75\%), similar in Italy (26 to $49 \%$ ), and lower in Austria (14\%). For Switzerland, there are no figures on the influenza vaccination coverage of children's an age group which is especially with regard to at-risk subjects, not adequately vaccinated worldwide.

Immunization of infants and young children requires two doses of vaccine spaced 1 or 2 months apart. However, current vaccines still do not result in a high, long-lasting protective immune response in this group, and vaccines with improved immunogenicity are clearly needed. However, reformulation of either whole-virus vaccines or subunit vaccines with new adjuvants or antigen delivery systems has been shown to greatly 
potentiate the immune response in animals ${ }^{[55]}$.

\section{Trafficking of Virosomes}

Trafficking of the virosomes to the cell surface has not been well characterized, but is presumed to occur via normal vesical maturation and exocytic process. The outcome is the nonlytic release of the vast majority of mature virions into the extracellular space. MHV and several other coronaviruses that can directly fuse with cells and there is a characteristic and rapidly detectable cytopathic effect of cell-cell fusion into multinucleated syncytia. The production of infectious virus continues even after the majority of cells are fused. Syncytia were recently reported as readout of $\mathrm{SCoV}$ receptor expression and cell infection. It is unknown whether $\mathrm{SCoV}$ is a respiratory virus or a pneumoenteric virus. This knowledge gap will be stymine efforts to develop a vaccine or drug against SCoV. Molecular biological studies have also identified several potential targets for antiviral drug discovery including viral binding and uncoating, replication, protein expression, processing and assembly, release cellular functions on which the virus depends on such as cholesterol synthesis membrane trafficking and autophagy ${ }^{[56]}$.

\section{Future Prospects of Virosomes}

Virosomes represent an innovative drug-delivery system for various biologically active molecules, especially nucleic acids or genes and for numerous indications. The outer surface of virosomes can be suitably modified to facilitate targeted drug delivery system. The comprehensive pharmacokinetic profile, bioavailability, clinical effects and stability should be studied thoroughly to ascertain their long-term reliability as a safe, effective, potent and affordable means for drug delivery and targeting.

\section{Conclusion}

Virosome is an emerging system of drug delivery and drug targeting on biological system. Improvisations of this tool will bring a new prospective and also open a new era in the modern pharmaceutical field and also human life too. Virosomes can be used to deliver an antigen in the administration by various routes like intranasal, intradermal, and intramuscular, depending on the aim of immunization without any side effects. An attractive feature of virosomes is the possibility to target them to selected cells using Fab fragments of monoclonal antibodies specific for the binding. Influenza virosomes can be a great tool for delivering antigens and molecules of different nature, such as proteins, peptides, plasmids, oligonucleoides and even drugs, to cells and due to their versatility and it is possible to exploit them in different scientific sectors. Virosomes could also be exploited as carriers for targeted drugs delivery and for immunomodulating molecules particularly in cancer therapy/treatment. All these features allow us to consider influenza virosomes as a promising model for antigen and/or unrelated molecules delivery and which could be helpful for the development of new vaccines or immunotherapy protocols that combine safety with immunogenicity and their applicability in different field of medical research.

\section{References}

1. Marc, P.G., Thomas,Cherian,. Yuri,Pervikar., et al. A review of vaccine research and development: human acute respiratory infections. (2005) Vaccine 23(50): 5708-5724.

2. Kay, M.A., Glorioso, J.C., Naldini, L. Viral vectors for gene therapy: the art of turning infectious agents into vehicles of therapeutics. (2001) Nat Med 7(1): 33-40.

3. Thomas, C.E., Ehrhardt, A., Kay, M.A. Progress and problems with the use of viral vectors for gene therapy. (2003) Nat RevGenet 4(5): 346-358.

4. Hacein, B.S., Von,Kalle, C, Schmidt, M., et al. LMO2-associated clonal $\mathrm{T}$ cell proliferation in two patients after gene therapy for SCID-X1. (2003) Science 302(5644):415-419.

5. Morral, N., O' Neal, W.K., Rice, K., et al. Lethal toxicity, severe endothelial injury, and a threshold effect with high doses of an adenoviral vector in baboons. (2003) Hum Gene Ther 13(1): 143-154.

6. Kafri, T., Morgan, D., Krahl, T., et al. Cellular immune response to adenoviral vector infected cells does not require de novo viral gene expression: implications for gene therapy. Proc. (1998) NatlAcadSci USA. 95(19): 11377-11382.

7. Schimidt, W.G.D., Schimidt, W.I.G. Non-viral and hybrid vectors in human gene therapy: an update. (2003) Trends Mol Med9(2): 67-72.

8. Kumar, V.V., Singh, R.S., Chaudhuri, A.Cationic transfection lipids in gene therapy: successes, set-backs, challenges and promises. (2003) Curr Med Chem 10(14): 1297-1306.

9. Crispin, R.D. Lipoplex-mediated delivery of nucleic acids: factors affecting in vivo transfection. (2004) JMol Med 82(9): 579-591.

10. Lima, M.C., Neves, S., Filipe, A., et al. Cationic liposomes for gene delivery: from biophysics to biological applications. (2003) Curr MedChem 10(14): 1221-1231.

11. Miller, A.D. The problem with cationic liposome/micelle-based non-viral vector systems for gene therapy. (2003) Curr Med Chem 10(14): 1195-1211.

12. Almeida, J.D., Edwards, D.C., Brand, C.M., et al. Formation of virosomes from influenza subunits and liposomes. (1975) Lancet2(7941): 899-901.

13. Bagai, S., Anu,P., Robert, B., et al. Hemagglutinin-neuraminidase enhances $F$ protein-mediated membrane fusion of reconstituted Sendai virus envelopes with cells. (1993) J Virol 67(6): 3312-3318.

14. Pöltl-Frank, F., Zurbriggen, R., Helg, A., et al.Use of reconstituted influenza virus virosomes as an immunopotentiating delivery system for a peptide-based vaccine. (1999) ClinExpImmunol 117(3): 496- 503. 15. Hunziker, I.P., Zurbriggen, R., Glueck, R., et al.Perspectives towards a peptide-based vaccine against hepatitis $C$ virus. (2001) MolImmunol 38(6): 475-84.

16. Mengiardi, B., Berger, R., Just, M., et al.Virosomes as carriers for combined vaccines. (1995) Vaccine 13(14): 1306-1315.

17. Mischler, R., Metcalfe, I.C.InflexalV a trivalent virosome subunit influenza vaccine: production. (2002) Vaccine 20(5): 17-23.

18. Rafael, M., Luyong, J., Kerstin, M., et al. Exploiting conformationally constrained peptidomimetics and an efficient human-compatible delivery system in synthetic vaccine design. (2001)Chembiochem 2(11): 838-843.

19. Rock, K.L., York, L.A., Saric, T., et al. Protein degradation and the generation of MHC class-I presented peptides. (2002) AdvImmunol 80: $1-59$.

20. Durrer, P., Glück, U., Spyr, C., et al. Mucosal antibody response induced with a nasal virosome-based influenza Vaccine.(2003) Vaccine21(27-30):4324-4334.

21. Both, G.W., Sleigh, M.J.,Cox, N.J., et al. Antigenic drift in influenza virus $\mathrm{H} 3$ hemagglutinin from 1968 to 1980: multiple evolutionary pathways and Sequential amino acid changes at key antigenic sites. (1983) J Virol 48(1): 52-60.

22. Carrat, F., Flahault, A.Influenza vaccine: the challenge of antigenic drift. (2007) Vaccine 25(39-40): 6852-6862.

23. WHO.Fact sheet Number 211(2003) Influenza. 
24. Metsikkö, K., van, M. G., Simons, K.Reconstitution of the fusogenic activity of vesicular stomatitis virus. (1986) EMBO J. 5(13): 3429-3435.

25. Petri, W.A., Wagner, R.R. Reconstitution into liposomes of the glycoprotein of vesicular stomatitis virus by detergent dialysis. (1979) J BiolChem 254(11): 4313-4316.

26. Glück, R., Mischler, R., Finkel, B., et al. Immunogenicity of new virosome influenza vaccine in elderly people. (1994) Lancet 344(8916): $160-163$.

27. Prem, N.G., Singh, P., Mishra, V. et al. Topical immunization:Mechanistic insight and novel delivery systems.(2004) Indian Journal of Biotechnology 3: 9-21.

28. Rajat, S., Mohd,Yasir. Virosomes: A Novel Carrier for Drug Delivery.(2010)International Journal of PharmTech Research, 2(4):23272339.

29. Huckriede, A., Bungener,L., terVeer, W., et al.Influenza virosomes: combining optimal presentationof hemagglutinin with immunopotentiating activity.(2003) Immunopotentiators in Modern Vaccines.21(9-10): 925-931.

30. Huckriede, A., Bungener, L., Stegmann, T., et al.The virosome concept for influenza vaccines. (2005) Vaccine 23(1): S26-38.

31. Kaneda, Y.Virosomes: evolution of the liposome as a targeted drug delivery system. (2000) Adv Drug Deliv Rev 43(2-3): 197-205.

32. Stegmann, T., Morselt, H.W., Booy, F.P., et al.Functional reconstitution of influenza virus envelopes. (1987) EMBO J 6(9): 2651-2659.

33. Bron, R., Ortiz, A., Dijkstra, J., et al.Preparation, properties, and applications of reconstituted influenza envelopes (virosomes). (1993) Methods in Enzimol 220: 313- 331

34. Pieter, S., Romke, B., Wilschut, J.Delivery of foreign substances to cells mediated by fusion-active reconstituted influenza virus envelopes (virosomes). (1993) J Liposome Res 3(3): 767-792.

35. Romke, B., Antonio, Ortiz., Wilschut, J.Cellular cytoplasmic delivery of a polypeptide toxin by reconstituted influenza-virus envelopes (virosomes). (1994) Biochem 33(31): 9110-9117.

36. Asghar, A., Hoorieh, S.,Masoumeh, T. K.,et al. Reconstruction of H3N2 influenza virus based virosome in-vitro. (2013) Iran J Microbiol 5(2): 166-171.

37. Felnerova, D., Viret, J.F., Glück, R., et al.Liposomes and virosomes as delivery systems for antigens, nucleic acids and drugs. (2004) CurrOpinBiotechnol 15(6): 518-529.

38. Maria, G.C.Applications of influenza virosomes as a delivery system. (2006) Human Vaccines 2(1): 1-7.

39. Schoen, P., Chonn, A., Cullis, P.R., et al.Gene transfer mediated by fusion protein hemagglutinin reconstituted in cationic lipid vesicles. (1999) Gene Ther. 6(5): 823-832.

40. Jonge, J., Holtrop, M., Wilschut, J., et al.Reconstituted influenza virus envelopes as an efficient carrier system for cellular delivery of small-interfering RNAs. (2005) Gene Ther 13(5): 400-411.

41. Dijkstra, J., Bron, R., Wilschut, J., et al.Activation of murine lymphocytes by lipopolysaccharide incorporated in fusogenic, reconstituted influenza virus envelopes (virosomes). (1996) J Immunol157(3): 1028- 1036.
42. Scardino, A., Correale, P., Firat, H., et al.In vivo study of the $G C / 90 I R I V$ vaccine for immune response and autoimmunity into a novel humanised transgenic mouse. (2003) Br J Cancer 89(1): 199-205.

43. Waelti, E., Wegmann, N., Schwaninger, R., et al.Targeting HER/ neu with antiratNeuvirosomes for cancer therapy. (2002) Cancer Res 62(2): 437-444.

44. Markus, S. M.,Annabelle, R., Francesca, B., et al. Induction of parasite growth inhibitory antibodies by a virosomal formulation of a peptidomimetic of loop I from domain III of Plasmodiumfalciparum apical membrane antigen 1. (2003) InfecImmun 71(8): 4749-4758.

45. Maria, G.C., Rinaldo, Z., Marcello, V., et al.Intranasal immunization with mumps virus DNA vaccine delivered by influenza virosomes elicits mucosal and systemic immunity. (2000) Virology 277(1): 111118.

46. Cusi, M.G., Del Vecchio, M.T, Terrosi, C., et al. Immune-Reconstituted Influenza Virosome containing CD40L gene enhances the immunological and protective activity of a carcinoembryonic antigen anti-cancer vaccine. (2005)J Immunol 174(11): 7210-7216.

47. Waelti, E.R, Glück,R. Delivery to cancer cells of antisense L-myc oligonucleotides incorporated in fusogenic, cationic-lipid-reconstituted influenza-virus envelopes (cationic virosomes).(1998) Int J Cancer 77(5): 728-733.

48. Cusi, M.G., Zurbriggen, R., Correale, P., et al. Influenza virosomes are an efficient delivery system for respiratory syncytial virus-F antigen inducing humoral and cell-mediated immunity. (2002) Vaccine 20(2930): 3436-3442.

49. Enrico, M., Pieter, S., Jan, W., et al. Targeting influenza virosomes to ovarian carcinoma cells. (2001) FEBS Letters. 509(1): 71-76.

50. Hunziker, I.P., Zurbriggen, R., Glueck, R., et al. Perspectives: towards a peptide- based vaccine against hepatitis C virus. (2001) MoIImmunol 38(6): 475-484.

51. Zurbriggen, R., Amacker, M., Kammer, A.R., et al. Virosome-based active immunization targets soluble amyloid species rather than plaques in a transgenic mouse model of Alzheimer's disease. (2005) J MolNeurosci 27(2): 157-166.

52. Moser, C., Amacker, M., Kammer, A.R., et al. Influenza virosomes as a combined vaccine carrier and adjuvant system for prophylactic and therapeutic immunizations. (2007) Expert Rev Vaccines 6(5): 711-721. 53. Priyanka, R., Gaurav, S. A. Virosomes: Novel vaccination technology. (2012)Int J PharmaSci Research 3(10): 3591-3597.

54. Erdmann, V.A., Barciszewski, J. DNA and RNA Nanobiotechnologies in medicine: Diagnosis and treatment of diseases. (2013) Springer Publisher. 87-356.

55. Schaad, U. B.,Buhlmann, U., Burger, R., et al. Comparison of immunogenicity and safety of a virosome influenza vaccine with those of a subunit influenza vaccine in pediatric patients with cystic fibrosis. (2000) Antimicrob Agents Chemother 44(5): 1163-1167.

56. Knobler, S., Mahmoud, A., Lemon, S., et al.Learning from SARS: Preparing for the Next Disease Outbreak. Workshop Summary. (2004) Institute of medicine of The National Academies, The national Academies Press, Washington, D.C. 1-376. 